\title{
Use of antithrombotic measures for stroke prevention in atrial fibrillation
}

\author{
I Perez, A Melbourn, L Kalra
}

\begin{abstract}
Objective-To evaluate appropriateness of antithrombotic use to prevent stroke in atrial fibrillation.

Design, patients-344 patients with atrial fibrillation, stratified by age, were assessed clinically for contraindications to anticoagulation and stroke risk. The use of warfarin and aspirin was compared with recommendations for anticoagulation derived from pooled clinical trial data.

Results-Low risk of stroke was seen in 47 (14\%) patients, moderate risk in 213 $(62 \%)$, and high risk in $84(24 \%)$ patients included in the sample (mean (SD) age 68.4 (17.2) years, $42 \%$ men). The proportion of patients requiring anticoagulation varied from $258 / 344(75 \%)$ to $72 / 344$ (21\%) depending upon criteria used, of whom $86 / 258(33 \%)$ and $36 / 72(50 \%)$ were receiving warfarin, respectively. Warfarin or aspirin were not being used in 124/297 $(42 \%)$ patients with moderate to high risk, whereas anticoagulation was being undertaken in $13 / 47(27 \%)$ patients at low risk of stroke. Antithrombotic use (warfarin or aspirin) was significantly less common in patients over 75 years of age, regardless of absence of contraindications and eligibility according to various criteria $(\mathrm{p}<0.001)$.

Conclusions-A clear need for anticoagulation using clinical criteria existed in about $25 \%$ of patients in atrial fibrillation presenting to medical clinics who were at high risk of stroke. Of these, only $50 \%$ of eligible patients were being anticoagulated. Appropriate anticoagulation needs to be based on risk assessment rather than age. Consensus is therefore needed on appropriate antithrombotic use in clinical practice.

(Heart 1999;82:570-574)
\end{abstract}

Keywords: stroke prevention; atrial fibrillation; warfarin; aspirin; antithrombotics

Adjusted dose warfarin has been shown to be effective in preventing stroke in patients with non-valvar atrial fibrillation. ${ }^{1}$ Overall stroke risk is reduced by $68 \%$ from $5 \%$ to $1.3 \%$ per year at the expense of increasing the risk of major bleeds to $1.1-2.3 \%$ and minor bleeds to $7.8-8.4 \%$ per year. ${ }^{12}$ However, the margin between expected benefit and harm is thin, especially in older patients and in those at low risk of stroke. ${ }^{3}$ There are clinical concerns that it may not be appropriate to anticoagulate all patients with atrial fibrillation, not only be- cause of the risks of anticoagulation but also because of the relative benefit in patients at low risk of stroke.

These concerns have been partially addressed by the development of clinical criteria for anticoagulation in patients with atrial fibrillation. ${ }^{14-6}$ However, clinical practice has been made difficult by the wide range of guidelines available currently and the variations in their content. ${ }^{7}$ Although several earlier studies have shown that antithrombotic measures are underused in patients with atrial fibrillation in clinical practice, these estimates vary according to the criteria used and many do not address the issue of appropriateness in patients being anticoagulated. ${ }^{8-11}$ The question of appropriateness becomes even more important as more recent reports show a trend towards increasing use of warfarin in patients with atrial fibrillation. ${ }^{12}$ As the decrease in risk is proportional to the individual patient's risk of stroke, there is a possibility that indiscriminate anticoagulation of patients in atrial fibrillation may reduce the effectiveness of anticoagulation in clinical practice. ${ }^{13}$

The appropriate approach to good clinical practice is one which balances the hazards of warfarin use with the expected reduction in stroke risk. ${ }^{14}$ Clinical trials suggest that it is possible to use clinical criteria for stratification of stroke risk, and those with the highest risk are most likely to benefit from anticoagulation..$^{11^{16}} \mathrm{It}$ is also possible to use these trials to identify a group of patients who are at low risk of stroke in whom the risks of anticoagulation may outweigh any benefits of stroke prevention. ${ }^{4-6}$

The objective of our study was to evaluate the appropriateness of anticoagulation in a sample of patients presenting to medical clinics over a one year period. The evaluation of appropriateness of clinical practice was determined by risk stratification based on criteria derived from pooled data from intervention studies. $^{14-6}$

\section{Methods}

This cross sectional study was undertaken in a suburban district general hospital, serving a population of 310000 . In accordance with national priorities in Britain, ${ }^{17}$ stroke prevention was a major strategic priority of the local health services and was supported by several initiatives in primary care. ${ }^{12}$ The district had well developed stroke and cardiovascular services and well organised anticoagulation clinics with free access to patients.

Subjects were recruited from patients under 90 years of age attending general medical clinics over a 12 month period. A retrospective 
review of notes was undertaken to identify patients with atrial fibrillation by screening general practitioner referral letters, hospital medical records, and ECGs in the notes. Patients were included if they were known to have atrial fibrillation for at least two months and had at least one subsequent clinic visit after the diagnosis of atrial fibrillation was confirmed. Patients receiving anticoagulation for other indications (such as pulmonary emboli, deep vein thrombosis) were excluded from the study.

Patients included in the study were reviewed by a research fellow, and an ECG was used to confirm the diagnosis of atrial fibrillation. A detailed clinical examination was undertaken which included assessments for the cause and duration of atrial fibrillation, risk of bleeding complications, and comorbidity or medication which may influence antithrombotic use. Patients were screened for vascular risk factors using a recommended protoco ${ }^{18}$ administered by the researchers. Laboratory investigations included full blood counts, coagulation profile, and renal and hepatic function tests. Patients were questioned about previous advice on warfarin or aspirin use, and medical records were examined for evidence to suggest that these issues had been discussed with patients or warfarin was considered inappropriate because of risks or issues of compliance.

Patients were stratified for stroke risk on clinical criteria derived from a modification of pooled data from clinical trials ${ }^{1315}$ and recommendations from existing guidelines. ${ }^{4-6} \mathrm{~Pa}-$ tients were considered to have low risk of stroke (annual risk 1\%) if under 65 years of age and with no history of embolism, hypertension, or diabetes. Moderate risk of stroke (annual risk $4 \%$ ) was considered to exist in: patients without previous embolism who were aged under 65 years but with a history of diabetes or hypertension; patients aged 65-75 years (regardless of hypertension or diabetes); or those aged over 75 years but with no history of hypertension or diabetes. Patients with a previous history of transient ischaemic attack or stroke (regardless of age), previous embolic episodes, or those over 75 years of age with hypertension or diabetes were considered to be at a high risk of stroke (annual risk 12\%). The high risk group also included patients with valve disease, cardiac prosthesis, uncompensated heart failure, cardiomyopathy, and known left ventricular dysfunction or intracardiac thrombus.

In the absence of accepted guidelines, the contraindications for anticoagulation were based on known contraindications to anticoagulation and exclusion criteria used in clinical trials. ${ }^{1315}$ Major contraindications to warfarin use included a history of bleeding from the gastrointestinal or genitourinary tract in the six months before diagnosis, known coagulation defects, thrombocytopenia or platelet dysfunction, haemorrhagic stroke, excessive alcoholic intake, recurrent falls, and poor drug or clinic compliance. Minor contraindications included dementia, uncontrolled hypertension, and use of non-steroidal agents.
Appropriate management of patients was derived from the findings of clinical trials and existing guidelines. ${ }^{13-615}$ Treatment with warfarin was considered to be appropriate management in patients at high risk of stroke and with no major contraindications to anticoagulation. The use of warfarin was considered inappropriate in patients at low risk of stroke or those who had major contraindications to anticoagulation. Appropriateness of anticoagulation use could not be established in patients at moderate risk of stroke or those with minor contraindications to anticoagulation because of the lack of consensus in this group. Treatment with either warfarin or aspirin was considered appropriate in this group.

The management of patients since the diagnosis of atrial fibrillation was compared against these criteria and the use of antithrombotics recorded. Further information was sought directly from patients or their medical records if they were at low risk but receiving warfarin, at high risk but not receiving warfarin, or at moderate or high risk but not receiving aspirin. The number of patients in whom anticoagulation had been discontinued and the reasons for discontinuation were recorded.

Patients were stratified according to age into three groups (under 65 years, $65-75$ years, and over 75 years) and antithrombotic use assessed in each group. Descriptive data are presented as mean, median, or proportion as appropriate. Analysis has been undertaken using the $\chi^{2}$ test for comparisons of independent proportions in more than two groups, except for data which include 0 as a value. This test has been chosen because of simplicity and robustness for such analyses. The bivariate association between antithrombotic treatment and use of warfarin and age, sex, level of stroke risk, individual vascular risk factors, and contraindications to anticoagulation was assessed using the $\chi^{2}$ test. Variables with $\mathrm{p}<0.1$ were entered into multiple regression analysis with step down deletion to investigate the independent effect of each variable.

\section{Results}

Of the 2457 patients seen in medical clinics over the study period, atrial fibrillation on ECG was present in $344(14 \%)$ patients. Valve disease, confirmed on echocardiography, was seen in 21 patients and cardiac prostheses in six patients. The mean age of patients was 68 years and there was a slight predominance of women (table 1). Nearly $60 \%$ of the patients included had additional risk factors, the most common being hypertension, previous stroke, and ischaemic heart disease (table 1). ${ }^{19} 20$ The median duration between the first diagnosis of atrial fibrillation and research assessment was 17.5 months (range 2-34 months). High risk of stroke was seen in a quarter of the patients, $90 \%$ of whom were over 65 years of age (table 2 ). In contrast, a low risk of stroke was seen in 47 patients, all of whom were under 65 years of age. The majority of patients had moderate risk of stroke $(213(62 \%))$, most of whom were aged $65-75$ years (table 2 ). 
Table 1 Demography and risk factor profile of 344 patients with atrial fibrillation

\begin{tabular}{ll}
\hline $\begin{array}{l}\text { Mean (SD) age (years) } \\
\text { Age range (years) }\end{array}$ & $68.4(17.2)$ \\
Male:female & $44-90$ \\
Risk factors & $146: 198$ \\
$\quad$ No risk factors & $123(36 \%)$ \\
Hypertension $^{19}$ & $148(43 \%)$ \\
Previous stroke $_{\text {Symptomatic ischaemic heart disease }}$ & $64(19 \%)$ \\
Diabetes mellitus $_{\text {Hypercholesterolaemia }}^{20}$ & $107(31 \%)$ \\
Smoking (current) & $48(14 \%)$ \\
Smoking (former) & $21(6 \%)$ \\
Alcohol > $>14$ units/week & $62(18 \%)$ \\
\hline
\end{tabular}

Major contraindications to anticoagulation were present in $39(11 \%)$ patients and 66 $(19 \%)$ patients had minor contraindications (table 3). A higher proportion of patients over the age of 75 years were ineligible for anticoagulation because of major contraindications compared with younger patients $(23 / 110$ $(20 \%)$ v 16/234 (7\%), p < 0.01). The most common major contraindications in younger patients were previous intracranial haemorrhage $(n=4)$ and excessive alcohol intake $(\mathrm{n}=4)$. In comparison, recurrent falls $(\mathrm{n}=8)$ and inability to comply with anticoagulation treatment $(n=10)$ were more common in older patients.

Anticoagulation was being undertaken in 36 $(50 \%)$ of the 72 patients at high risk of stroke who did not have a major contraindication to warfarin use (table 3). Nearly a quarter of patients in this group were not receiving any antithrombotic treatment (warfarin or aspirin) despite the high risk. This was particularly true for patients over 75 years of age $(p<0.001)$, a group in which $80 \%$ of patients with no contraindications were not being anticoagulated and only a quarter were receiving aspirin. Warfarin was being used in 50/186 $(27 \%)$ of eligible patients at moderate risk of stroke. Despite a higher than average risk of stroke,

Table 2 Age stratified stroke risk in 344 patients in atrial fibrillation

\begin{tabular}{lrrrllll}
\hline $\begin{array}{l}\text { Age } \\
\text { (years) }\end{array}$ & \multicolumn{1}{c}{$n$} & Male & Female & Other risks & Low & Moderate & High \\
\hline$<65$ & 63 & 34 & 29 & $16(14 \%)$ & $47(75 \%)$ & $11(17 \%)$ & $5(8 \%)$ \\
$65-75$ & 171 & 87 & 84 & $115(67 \%)$ & - & $126(74 \%)$ & $45(26 \%)$ \\
$>75$ & 110 & 48 & 62 & $83(75 \%)$ & - & $76(69 \%)$ & $34(31 \%)$ \\
Total & 344 & 169 & 175 & 214 & $47(14 \%)$ & $213(62 \%)$ & $84(24 \%)$ \\
\hline
\end{tabular}

Table 3 Eligibility and antithrombotic use in patients with atrial fibrillation stratified according to risk

\begin{tabular}{|c|c|c|c|c|c|c|}
\hline \multirow[b]{2}{*}{ Age (years) } & \multirow[b]{2}{*}{$n$} & \multicolumn{2}{|c|}{ Contraindications } & \multirow[b]{2}{*}{ Warfarin } & \multirow[b]{2}{*}{ Aspirin } & \multirow[b]{2}{*}{ No treatment } \\
\hline & & Major & Minor & & & \\
\hline \multicolumn{7}{|c|}{ Low risk (annual rate $1 \%$ ) } \\
\hline$<65$ & 47 & 1 & $8(17 \%)$ & $13 / 47(27 \%)$ & 5 & $29 / 47(62 \%)$ \\
\hline Total & 47 & 1 & $8(17 \%)$ & $13 / 47(27 \%)$ & 5 & $29 / 47(62 \%)$ \\
\hline \multicolumn{7}{|c|}{ Moderate risk (annual rate $4 \%$ ) } \\
\hline$<65$ & 11 & 1 & 1 & $5 / 10(50 \%)$ & 2 & $4 / 11(36 \%)$ \\
\hline $65-74$ & 126 & 9 & 23 & $43 / 116(37 \%)$ & 49 & $34 / 126(27 \%)$ \\
\hline$>75$ & 76 & 16 & 17 & $2 / 60(3 \%)$ & 11 & $63 / 76(83 \%)$ \\
\hline Total & 213 & $26(12 \%)$ & $41(19 \%)$ & $50 / 186(27 \%)$ & 62 & $101 / 213(47 \%)$ \\
\hline \multicolumn{7}{|c|}{ High risk (annual risk 12\%) } \\
\hline$<65$ & 5 & 0 & 1 & $4 / 5(80 \%)$ & 1 & 0 \\
\hline $65-74$ & 45 & 5 & 8 & $27 / 40(67 \%)$ & 11 & $7 / 45(16 \%)$ \\
\hline$>75$ & 34 & 7 & 8 & $5 / 27(19 \%)$ & 13 & $16 / 34(47 \%)$ \\
\hline Total & 84 & $12(14 \%)$ & $17(20 \%)$ & $36 / 72(50 \%)$ & 25 & $23 / 84(27 \%)$ \\
\hline
\end{tabular}

Patients receiving warfarin represented as a percentage of patients with no contraindications and eligible for warfarin use. nearly half of these patients were receiving neither warfarin nor aspirin. Regular anticoagulation for stroke prevention was being undertaken in $13(27 \%)$ patients at low risk of stroke, despite no other indication. Five (11\%) patients were receiving aspirin. No treatment had been prescribed in $29(62 \%)$ patients.

Anticoagulation was being undertaken in three patients with major contraindications. Of these, one patient was at low and two patients at moderate stroke risk. None of these patients had suffered an adverse event between starting treatment and assessment. Anticoagulation had been discontinued in five patients at high risk of stroke because of complications. The treatment had been discontinued in four patients at low risk (deemed inappropriate 2; poor compliance 2), and in seven patients at moderate risk of stroke (poor compliance 2; bleeding episode 1 ; anaemia of unknown origin 2; unknown reasons 2).

Multiple regression analysis showed that age had a significant effect on the level of stroke risk which was independent of sex and other vascular risk factors in this patient group. There was a strong positive association between the level of risk and treatment (b $\left.=0.543, p<0.001, r^{2}=0.64\right)$ as well as between the level of risk and use of warfarin for the whole patient group $(b=0.324, p<0.01$, $r^{2}=0.72$ ). Advancing age was negatively associated with the use of warfarin regardless of the level of risk $(b=-1.012, p<0.0001$, $\left.r^{2}=0.49\right)$.

\section{Discussion}

There have been many studies which have looked at the use of anticoagulation in atrial fibrillation without addressing the issue of appropriateness according to risk stratification or the ease of access to anticoagulation services. ${ }^{8-11}$ Risk stratification in determining the appropriateness of anticoagulation may be an important issue because a recent analysis of the original trials on anticoagulation in atrial fibrillation has shown that the margin between expected benefit and harm may be uncomfortably thin. ${ }^{16}$ The reduction in annual incidence of major stroke was less than $1 \%$ (previously thought to be about $2.5-3 \%$ ) with an increase in the risk of major bleeding by nearly $2 \% .{ }^{16}$ Similarly, access to anticoagulation services may be a limiting factor and there may be differences between settings where this service is freely available from those where access is restricted.

This study shows that a clear need for anticoagulation using clinical criteria existed in about $25 \%$ of patients in atrial fibrillation presenting to medical clinics who were at high risk of stroke. Of these, only $50 \%$ of eligible patients were being anticoagulated despite prioritisation of stroke prevention in national and local health programmes ${ }^{12}$ and a freely available anticoagulation service. More than a quarter of patients were receiving no antithrombotic treatment despite a high risk of stroke. On the other hand nearly a quarter of the patients at low risk of stroke, in whom the least benefit was expected, were receiving warfarin. Anticoagu- 
lants were used in only one in five eligible patients over 75 years of age who were at high risk of stroke and most likely to benefit from this intervention. These figures are more alarming in view of the fact that sampling in this study was undertaken in a highly focused group of patients presenting to secondary care and may be not be representative of practice in the wider community.

The largest group of patients in this study comprised those at moderate risk of stroke. Literature is divided about the benefits of anticoagulation in this patient group, with some guidelines suggesting benefits of anticoagulation ${ }^{36}$ while others favour the use of antiplatelet agents. ${ }^{45}$ Decision making in clinical practice is further complicated by the emergence of new criteria for anticoagulation (stroke prevention in atrial fibrillation investigators, American College of Chest Physicians consensus conference ${ }^{21}$ ) which may often differ in content or emphasis. Whereas most criteria seem to be reasonably consistent in identifying those at high or low risk in clinical terms, they are not clear for those with moderate risk, which makes it difficult to assess appropriateness of anticoagulation in this group. If anticoagulation were to be considered appropriate in patients at moderate risk, this study suggests that anticoagulation will be required in 258/ $344(75 \%)$ patients, of whom only $86(33 \%)$ were receiving the intervention. These figures are in keeping with another recent study on eligibility for anticoagulation in the community based on this assumption. ${ }^{22}$ On the other hand, if anticoagulation was not indicated in this group of patients, only $72 / 344(21 \%)$ would require warfarin and it could be argued that $63 / 344(18 \%)$ patients were being exposed unnecessarily to the risks of anticoagulation in this study.

It may be possible to define eligibility for anticoagulation better by using echocardiographic criteria in addition to clinical criteria in assessing risk. ${ }^{23}$ Routine echocardiography to determine thromboembolic risk in large numbers of patients with atrial fibrillation (6-10\% of the population over 75 years of age ${ }^{24}$ ) has practical limitations, considering the variations in the availability of echocardiography in Great Britain. ${ }^{25}$ The value of echocardiography also depends on how frequently echocardiography is the only method that will identify risk. A recent study suggests that all patients with atrial fibrillation who have any other clinical risk factor will require anticoagulation, and echocardiography had little additional value in their management. ${ }^{22}$ The study showed that echocardiography was likely to influence treatment decisions only in younger patients who had no clinical risk factors. ${ }^{22}$ If confirmed, these findings have important implications for clinical practice. It may be possible to rationalise the use of echocardiography to selected patients with moderate or low risk of stroke in whom it is likely to influence clinical decisions. It also implies that the majority of patients in the moderate risk group should be anticoagulated, suggesting that the underuse of warfarin in general practice is greater than identified by this study.

The use of warfarin was significantly influenced by age, with increasing age having an adverse effect on anticoagulant use independent of level of stroke or bleeding risk. These findings contrast with surveys which show a high level of physician agreement on the effectiveness of anticoagulation and warfarin use, even in elderly people. ${ }^{26}$ However, they are in keeping with the practice observed in other studies which show a reluctance to use warfarin in older age groups regardless of the level of anticoagulation risk. ${ }^{27}$ This is because of the widespread perception that elderly patients may not be suitable candidates for anticoagulation for unspecified reasons. Many of these unspecified reasons such as dementia, poor compliance, recurrent falls, and use of nonsteroidal agents are quantifiable and are known contraindications to anticoagulation. Despite considering such patients as ineligible for anticoagulation in this study, there continued to be a large proportion of eligible patients who were not receiving warfarin or aspirin for stroke prevention. It is well known that a higher proportion of elderly people will have contraindications to warfarin use $(20 \% v 7 \%$ in this study), but this should not be the reason why a large proportion of eligible elderly people are denied appropriate treatment. The message from this and other studies is that age alone should not be a major determinant of eligibility for anticoagulation in patients with atrial fibrillation. Clinical emphasis should be on undertaking objective risk/benefit assessment regardless of age to identify patients most likely to derive the greatest benefit from regular warfarin use.

The study suggests that the lack of generally agreed criteria for anticoagulation rather than awareness of the benefits of anticoagulation or the availability of anticoagulation services may be responsible for the low treatment levels in patients with atrial fibrillation. The implementation of policies on anticoagulation in patients with atrial fibrillation for stroke prevention is made difficult by the imprecise estimation of the proportion of patients who may benefit from anticoagulation and the level of services required to initiate and monitor anticoagulation. ${ }^{28}$ Consensus on criteria for anticoagulation and clear guidelines for clinical practice which are consistent and universally accepted will facilitate the determination of appropriateness and allow precise quantification of service resource needs. There is little doubt that wider use of appropriate anticoagulation in atrial fibrillation will improve the efficacy and cost effectiveness of stroke prevention, and the costs of expansion of anticoagulation services will be repaid by the costs of strokes averted. ${ }^{29}$

1 Atrial Fibrillation Investigators. Risk factors for stroke and efficacy of antithrombotic treatment in atrial fibrillation: enclys of analysis of pooled data from five randomised controlled 2 Koefoed BG, Gullov AL, Petersen P. Prevention of Koefoed BG, Gullov AL, Petersen P. Prevention of
thromboembolic events in atrial fibrillation. Thromb thromboembolic events
Haemost 1997;78:377-81. 
3 Stroke Prevention in Atrial Fibrillation Investigators. Bleeding during antithrombotic therapy in patients with atria
fibrillation. Arch Intern Med 1996;156:409-16.

4 Lip GY, Lowe GD. ABC of atrial fibrillation. Antithrombotic treatment for atrial fibrillation. BMf 1996;312:45-9.

5 Laupacis A, Albers G, Dalen J, et al. Antithrombotic therapy in atrial fibrillation. Fourth ACCP consensus conference on antithrombotic therapy. Chest 1995;108(suppl 4):352S9S.

6 Matchar DB, McCroy DC, Barnett HJM, et al. Guidelines for medical treatment for stroke prevention. Ann Intern Med 1994;121:54-5.

7 Thomson RG, McElroy H, Sudlow M. Guidelines on anticoagulant treatment in Great Britain: variation in content and implication for treatment. BMf 1998;316:509-13.

8 Bath PMW, Prasad A, Brown MM, et al. Survey of use of anticoagulation in patients with atrial fibrillation. $B M \mathcal{F}$ 1996;312:45-9.

9 Albers GW, Yim JM, Belew KM, et al. Status of antithrombotic therapy for patients with atrial fibrillation in botic therapy for patients with atrial fibrillation
university hospitals. Arch Intern Med 1996;156:2311-16.

10 Whittle J, Wickenheiser L, Venditti LN. Is warfarin being underused in the treatment of elderly persons with atrial Antern Med 1997;157:451-5.

11 Brass LM, Krumholz HM, Scinto JM, et al. Warfarin use among patients with atrial fibrillation. Stroke 1997;28 2382-9.

12 Kalra L, Perez I, Melbourn A. Stroke risk management: changes in mainstream practice. Stroke 1998;29:53-7.

13 Gottlieb LK, Salem SS. Anticoagulation in atrial fibrillation: does effectiveness in clinical trials translate into effective practice? Arch Intern Med 1994;154:1945-53.

14 Glasziou PP, Irwig LM. An evidence based approach to individualising treatment. BMF 1995;311:1356-9.

15 Stroke Prevention in Atrial Fibrillation Investigators. Adjusted dose warfarin versus low intensity fixed dose warfarin plus aspirin for high risk patients with atrial farin plus aspirin for high risk

16 Green CJ, Hadorn DC, Bassett K, et al. Anticoagulation in chronic non-valvular atrial fibrillation: a critical appraisal and meta-analysis. Can f Cardiol 1997;13:811-15.
17 Department of Health. Health of the nation: a strategy for England and Wales. London: HMSO, 1992.

18 O'Mahoney PG, Robson R, Rodger H, et al. Validation of a population based questionnaire to assess prevalence of stroke. Stroke 1995;26:1334-7.

19 Sever P, Beevers G, Bulpitt C, et al. Management guidelines in essential hypertension: report of the second working party of the British Hypertension Society. BMF 1993;306: 983-7.

20 Betteridge DJ, Dodson PM, Durrington PN, et al. Management of hyperlipidaemia: guidelines of the British Hyperlipidaemia Association. Postgrad Med f 1993;69:359-69.

21 Laupacis A, Albers G, Dalen J, et al. Antithrombotic therapy in atrial fibrillation. Chest 1998;114:5795-895.

22 Sudlow M, Thomson R, Thwaites B, et al. Prevalence of atrial fibrillation and eligibility for anticoagulants in the community. Lancet 1998;352:1167-71.

23 Stroke Prevention in Atrial Fibrillation Investigators. Predictors of thromboembolism in atrial fibrillation: echocardiographic features of patients at risk. Ann Intern Med 1992;116:6-12.

24 Feinberg WM. Anticoagulation for prevention of stroke. Neurology 1998;51(suppl 3):S20-2.

25 Goldstein LB, Farmer A, Matchar DB. Primary care physician reported secondary and tertiary stroke prevention practices. Stroke 1997;28:746-51.

26 McCrory DC, Matchar DB, Samsa G, et al. Physician attitudes about anticoagulation for non-valvular atrial fibrillation in the elderly. Arch Intern Med 1995;155:27781

27 Whittle J, Wickenheiser L, Venditti LN. Is warfarin underused in the treatment of elderly persons with atrial fibrillation? Arch Intern Med 1997;157;441-5.

28 Sudlow CM, Rodger H, Kenny RA, et al. Service provision and use of anticoagulants in atrial fibrillation. $B M F$ 1995;311:558-60.

29 Gustafsson WG, Asplund K, Britton M, et al. Costeffectiveness of primary stroke prevention in atrial fibrillation: Swedish national perspective. BMF 1992;305: $1457-60$. 